Introduction/Background Endometrial cancer (EC) is currently the most frequent cancer affecting the female genital system. The treatment of choice is represented by surgery, which consists of bilateral hysterectomy and adnexectomy with lymphadenectomy (L) according to risk factor for recurrence. The systematic lymphadenectomy procedure is often associated with postoperative complications, such as lymphedema, lymphocysts, lymphorrhoea. Recently, sentinel lymph node mapping have overcome the complications associated with $\mathrm{L}$, though there is limited access to this tecnique. The aim of the study was to evaluate the role of radiomic analysis of pelvic adipose tissue at CT in predicting the incidence of post-oeprative complications of $\mathrm{L}$.

Methodology Consecutive patients who underwent surgical treatment of endometrial cancer at Careggi University Hospital between January 2016 and Decemeber 2019 were enrolled. Only patients underwent to pelvic lymphadenectomy were enrolled. Exclusion criteria were bulky nodes at the preoperative imagings. Staging CT images were used fot the radiomic analysis; pelvic adipose tissue was identified and segmented, so the images were imported to the 3D Slicer software. Subsequently, the extractions of the three radiomics features (busyness, flatness, elongation) of the rea of interest were carried out.

Results Twenty seven patients were enrolled. Five patients developed post-operative complications. The value of Busyness, Flatness and elongation correlated with postoperative complications $(\mathrm{p}=0.04, \mathrm{p}=0.021, \mathrm{p}=0.03$, respectively).

Conclusion Our preliminary study shows that radiomic might be useful to predict whether a patient will develop any complications associated with the lymphadenectomy. Consequently pre-operative imaging might be used also to select which patient benefit the most from sentinel node study instead of L. Disclosures The authors have no conflicts of interest to declare.

\section{ONCOLOGICAL OUTCOME OF SENTINEL LYMPH NODE MAPPING OR COMPREHENSIVE SURGICAL STAGING IN PATIENTS WITH NODE-NEGATIVE INTERMEDIATE-RISK ENDOMETRIAL CANCER}

Franziska Siegenthaler, Sara Imboden, Michael Mueller. University Hospital Bern, Inselspital; Department of Obstetrics and Gynecology

\subsection{6/ijgc-2020-ESGO.81}

Introduction/Background The role of lymphadenectomy in surgical staging for endometrial cancer remains controversial. The standard of care - consisting of a pelvic and para-aortic lymphadenectomy (LND) - has failed to show survival advantage while leading to an increased peri- and postoperative morbidity. Sentinel lymph node (SLN) mapping has gained popularity, offering a compromise between no nodal staging and complete LND. Multiple studies have demonstrated high detecection rates and negative predictive values of SLN mapping with near-infrared fluorescence imaging and indocyanine green (ICG) in endometrial cancer. However, the literature contains limited data on its safety and oncological outcomes. Aim of this study is to evaluate the oncological outcome of SLN mapping in patients with intermediate-risk endometrial cancer.

Methodology In a retrospective, single-center study, we investigated the oncological outcome of patients with stage I intermediate-risk endometrial cancer who underwent surgical staging at our institution between February 2013 and July 2020.
Results Out of a total number of 306 patients with endometrial cancer, 57 patients were diagnosed with node-negative intermediate-risk endometroid endometrial cancer (FIGO IA grade 3, FIGO IB grade 1 or 2). All patients were treated with laparoscopic hysterectomy and bilateral salpingo-oophorectomy with ICG SLN mapping. 31 patients additionally underwent comprehensive surgical staging (four systematic pelvic lymphadenectomies and 27 pelvic and para-aortic lymphadenectomies, LND group). Mean follow up time was 38.0 months. Adjuvant treatment consisted of vaginal brachytherapy in 49 patients, additional chemotherapy in four patients and no adjuvant treatment in eight patients. Between the two cohorts, there were no differences in age or BMI. The mean number of lymph nodes removed (4.04 vs. 45.5), the duration of the surgical procedure (131.3 vs. 287 minutes) as well as the intraoperative blood loss (101.9 vs. $258.1 \mathrm{ml})$ were significantly higher in the LND group $(\mathrm{p}=0.000,0.000$ and 0.026 , respectively). Recurrence rates $(7.7 \%$ SLN, $9.7 \%$ LND, $\mathrm{p}=0.585)$ and death due to disease $(3.8 \%$ SLN, 3.2\% LND, $\mathrm{p}=0.709$ ) were similar between the two groups. Further on, there was no statistically significant difference in overall and recurrence free survival for patients with SLN mapping only compared to the LND cohort $(\mathrm{p}=0.541$ and 0.480 , respectively).

Conclusion In our cohort, the use of ICG SLN mapping alone did not impair oncological outcome compared to a complete lymphadenectomy. It therefore might provide an efficient alternative of nodal staging with less morbidity in intermediate-risk endometrial cancer patients. However, prospective studies on larger numbers of patients are needed to confirm our findings.

Disclosures No disclosures.

\section{SENTINEL LYMPH NODE MAPPING WITH INDOCYANINE GREEN IN ROBOTIC-ASSISTED LAPAROSCOPIC SURGERY FOR EARLY ENDOMETRIAL CANCER: A POPULATION- BASED COHORT STUDY}

${ }^{1}$ Anastasios Tranoulis, ${ }^{2}$ Hebatallah Awad, ${ }^{2}$ Christina Thomson, ${ }^{2}$ Amy Fisher, ${ }^{2}$ Jeremy Twigg. ${ }^{1}$ The Pan-Birmingham Gynaecological Oncology Centre, Sandwell and West Birmingham NHS Trust, Birmingham; ${ }^{2}$ The James Cook University Hospital

\subsection{6/ijgc-2020-ESGO.82}

Introduction/Background The sentinel lymph node (SLN) biopsy may have a key role in endometrial cancer (EC), as the therapeutic effect of lympadenectomy per se remains a field of contention. The aim of this study was to analyse our experience using indocyanine green for SLN mapping in a minimally robotic-assisted laparoscopic approach with Da Vinci Si near-infrared (NIR) fluorescence imaging system.

Methodology This is a retrospective population-based cohort study of prospectively collected data, spanning the period from January 2015 to March 2020. A total of 172 women, who underwent robot-assisted laparoscopic surgery with the Da Vinci Si Surgical System with NIR imaging and indocyanine (ICG) fluorescence detection for early stage EC, were enrolled. Cervical injection with ICG $(2 \mathrm{ml})$ was performed for all patients. Baseline demographics, peri-operative and follow-up data were prospectively collected. We calculated the unilateral and bilateral detection rate. Possible correlations amongst the variables were examined using the Spearman's correlation coefficient (rho), whilst multivariate logistic 